\title{
Meningioma causing gabapentin-responsive secondary SUNCT syndrome
}

\author{
Anna Kutschenko • David Liebetanz
}

Received: 17 March 2010/Accepted: 11 April 2010/Published online: 29 April 2010

(C) The Author(s) 2010. This article is published with open access at Springerlink.com

\begin{abstract}
Short-lasting unilateral neuralgiform headache with conjunctival injection and tearing (SUNCT) is one of the rarest and most serious headache disorders. Cases of symptomatic SUNCT syndromes are reported, which demonstrate that brain imaging is very important for diagnosis. In this study, we describe the first case of secondary SUNCT syndrome caused by a meningioma. So far, a clearly effective therapy for SUNCT syndrome has not been known. In this case, however, SUNCT was completely responsive to gabapentin. This underlines that this drug is worthy of being considered as a potential therapeutic option in the treatment of SUNCT syndrome.
\end{abstract}

Keywords Secondary headache disorders .

SUNCT syndrome · Gabapentin · Meningioma

$\begin{array}{ll}\text { Abbreviations } \\ \text { SUNCT } & \begin{array}{l}\text { Short-lasting unilateral neuralgiform headache } \\ \text { with conjunctival injection and tearing }\end{array} \\ \text { SUNA } & \begin{array}{l}\text { Short-lasting unilateral neuralgiform headache } \\ \text { attacks with cranial autonomic features }\end{array} \\ \text { MRI } & \text { Magnetic resonance imaging }\end{array}$

A. Kutschenko $(\square)$. D. Liebetanz Department of Clinical Neurophysiology, University Medical Centre Göttingen, Robert-Koch-Strasse 40, 37099 Göttingen, Germany e-mail: anna.kutschenko@med.uni-goettingen.de

\section{Introduction}

Short-lasting unilateral neuralgiform headache with conjunctival injection and tearing (SUNCT) is an extremely rare form of headache. It is characterized by short attacks of unilateral neuralgiform severe pain accompanied by autonomic symptoms such as tearing, rhinorrhea and conjunctival injection [1-3]. SUNCT is a subset of SUNA (short-lasting unilateral neuralgiform headache attacks with cranial autonomic features) that shows similar headache attacks, but different cranial autonomic symptoms [4]. SUNCT syndrome is, as well as cluster headache and paroxysmal hemicranias, classified as a trigeminal autonomic cephalalgia (TAC). A possible pathophysiological mechanism of these headache disorders is a hypothalamic activation as shown by functional MRI studies [3, 5].

However, several symptomatic SUNCT syndromes caused by intracerebral tumors and non-tumorous lesions are reported. For instance, prolactinomas [6] and vascular malformations [7] have been described. So far, meningiomas have not been reported.

A definitely effective drug therapy is not known. Placebo-controlled studies do not exist. However, there are some case reports that demonstrate the potential efficacy of lamotrigine, gabapentin, topiramate and intravenous lidocaine $[2,3,8]$.

We report a case of secondary SUNCT syndrome caused by a meningioma fully responsive to gabapentin.

\section{Case report}

An 81-year-old woman presented with a 5-month history of headache. She had not presented earlier because she intended to commit suicide due to severe facial pain. The patient 
Fig. 1 Magnetic resonance imaging of the fronto-temporal meningioma. a MRI shows the left-sided fronto-temporal infiltrative growing T2-hyperintense tumor (asterisk) and (b, c) a T1-contrast medium enhancement (arrow). Note the meningeal tail sign, which is pathognomonic for meningiomas (arrow)
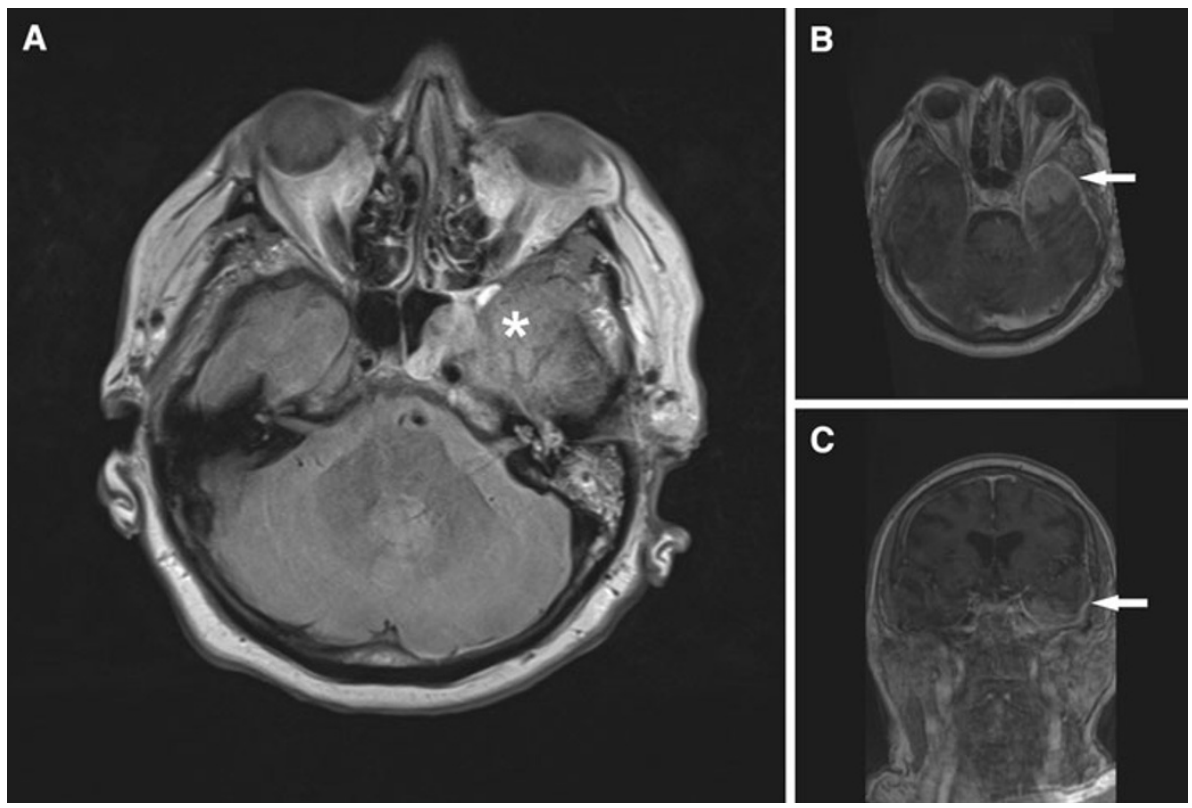

characterized the headache as an excruciating left-sided pain with a stabbing and neuralgiform quality, which spread out from the left forehead to the left temple and cheek. The very short-lasting attacks occurred about 60 times daily with a common occurrence at night. Triggers could not be found out. The attacks were accompanied by lacrimation, conjunctival injection, nasal congestion and rhinorrhea.

Physical examination showed a slight protrusion of the left eyeball, hypesthesia of the left side of the face and a left hearing impairment.

Brain MRI (magnetic resonance imaging) demonstrated an infiltrative growing left extraaxial fronto-temporal mass of about $2 \times 3.4 \times 3.5 \mathrm{~cm}$ that was T2 hyperintense and $\mathrm{T} 1$ isointense. It showed furthermore a clear contrast medium enhancement with a meningeal tail sign that was pathognomonic for meningiomas (see Fig. 1).

Because of her comorbidity consisting of cardiovascular diseases, the patient denied surgery. Therefore, we decided to start a symptomatic therapy. With a daily dosage of $1,800 \mathrm{mg}$ gabapentin, the patient was completely free of complaints.

One month later, the patient presented again with severe headache. In the meantime, the pain medication was changed from gabapentin to an opioid due to serial rib fracture. As the opioid was not effective in the treatment of the patient's headache, gabapentin therapy was reestablished. The patient was again asymptomatic with a daily dosage of $1,800 \mathrm{mg}$ gabapentin.

\section{Discussion}

The patient fulfills the criteria of the International Headache Society for SUNCT syndrome [1, 2]. The side and symptoms of this SUNCT syndrome are in accordance with the location of the meningioma. That is why we assume a causal relation. However, this causality could not be confirmed in the end because the patient refused an operation due to her comorbidity. To our knowledge, this is the first case reporting a SUNCT syndrome probably caused by a meningioma. Since a lot of other symptomatic SUNCT syndromes are described [6, 7], this case underlines that brain MRI is essential for the diagnosis of SUNCT syndrome.

Because of the favorable side effect profile, we settled for a symptomatic therapy with gabapentin. In contrast to pregabalin, its dosage can be increased more rapidly. Gabapentin has an inhibitory effect on voltage-gated calcium channels and is used as an antiepileptic drug as well as in the treatment of neuropathic pain. In this case, it was shown that the patient's neuralgiform pain was completely reactive to gabapentin, but not to opioids.

This case demonstrates that it is worth giving gabapentin a trial in the treatment of SUNCT syndrome.

\section{Conflict of interest None.}

Open Access This article is distributed under the terms of the Creative Commons Attribution Noncommercial License which permits any noncommercial use, distribution, and reproduction in any medium, provided the original author(s) and source are credited.

\section{References}

1. Sjaastad O, Saunte C, Salvesen R, Fredriksen TA, Seim A, Roe OD, Fostad K, Lobben OP, Zhao JM (1989) Shortlasting unilateral neuralgiform headache attacks with conjunctival injection, tearing, sweating, and rhinorrhea. Cephalalgia 9:147-156 
2. May A, Leone M, Afra J, Linde M, Sandor PS, Evers S, Goadsby PJ, EFNS Task Force (2006) EFNS guidelines on the treatment of cluster headache and other trigeminal autonomic cephalalgias. Eur J Neurol 13:1066-1077

3. Cohen AS (2007) Short-lasting unilateral neuralgiform headache attacks with conjunctival injection and tearing. Cephalalgia 27:824-832

4. Cohen AS, Matharu MS, Goadsby PF (2006) Short-lasting unilateral neuralgiform headache attacks with conjunctival injection and tearing (SUNCT) or cranial autonomic features (SUNA) - a prospective clinical study of SUNCT and SUNA. Brain 129:2746-2760
5. Matharu M, May A (2008) Functional and structural neuroimaging in trigeminal autonomic cephalalgias. Curr Pain Headache Rep 12:132-137

6. Massiou H, Launay JM, Levy C, El Amrani M, Emperauger B, Bousser MG (2002) SUNCT syndrome in two patients with prolactinomas and bromocriptine-induced attacks. Neurology 58:1698-1699

7. Mondejar B, Cano EF, Perez I, Navarro S, Garrido JA, Velasquez JM, Alvarez A (2006) Secondary SUNCT syndrome to a variant of the vertebrobasilar vascular development. Cephalalgia 26:620-622

8. Etemadifar M, Maghi AH, Ghasemi M, Chitsaz A, Kaji Esfahani M (2008) Efficacy of gabapentin in the treatment of SUNCT syndrome. Cephalalgia 28:1339-1342 